\title{
A Tale of Two Models: Mouse and Zebrafish as Complementary Models for Lymphatic Studies
}

\author{
Jun-Dae Kim, and Suk-Won Jin*
}

\begin{abstract}
Lymphatic vessels provide essential roles in maintaining fluid homeostasis and lipid absorption. Dysfunctions of the lymphatic vessels lead to debilitating pathological conditions, collectively known as lymphedema. In addition, lymphatic vessels are a critical moderator for the onset and progression of diverse human diseases including metastatic cancer and obesity. Despite their clinical importance, there is no currently effective pharmacological therapy to regulate functions of lymphatic vessels. Recent efforts to manipulate the Vascular Endothelial Growth Factor-C (VEGFC) pathway, which is arguably the most important signaling pathway regulating lymphatic endothelial cells, to alleviate lymphedema yielded largely mixed results, necessitating identification of new targetable signaling pathways for therapeutic intervention for lymphedema. Zebrafish, a relatively new model system to investigate lymphatic biology, appears to be an ideal model to identify novel therapeutic targets for lymphatic biology. In this review, we will provide an overview of our current understanding of the lymphatic vessels in vertebrates, and discuss zebrafish as a promising in vivo model to study lymphatic vessels.
\end{abstract}

\section{INTRODUCTION}

Since its first description in the $18^{\text {th }}$ century, diverse physiological events have been attributed to lymphatic vessels (Alitalo, 2011; Kerjaschki, 2014). For instance, lymphatic vessels regulate interstitial fluid homeostasis, facilitate the absorption of lipids, and provide a conduit for immune cells as well as metastasizing tumors (Achen et al., 2005; Wang and Oliver, 2010). Dysfunctions of lymphatic vessels lead to the swelling of extremities in humans, collectively referred to as lymphedema (Connell et al., 2013; Murdaca et al., 2012). While primary lymphedema, caused by genetic mutations, is relatively rare, secondary lymphedema,

Yale Cardiovascular Research Center, Section of Cardiovascular Medicine, Department of Internal Medicine, Yale University School of Medicine, New Haven, CT 06511, USA

${ }^{*}$ Correspondence: suk-won.jin@yale.edu

Received 29 April, 2014; accepted 2 May, 2014; published online 23 May, 2014

Keywords: lymphatic endothelial cell (LEC), lymphatic vessels, lymphedema, zebrafish which is induced by obstruction of lymphatic vessels, affects as many as 140-250 million people worldwide (Zuther and Norton, 2012). While substantial information on the development, morphogenesis, and maintenance of lymphatic vessels is available, how lymphatic vessels form during development remains poorly understood, partly due to the limitation of the model system of choice for lymphatic research. Zebrafish, which are amenable for time-lapse analysis and genetic manipulation (Gore et al., 2012; Wilkinson and van Eeden, 2014), have recently emerged as an important in vivo model system to investigate development and maintenance of lymphatic vessels (Koltowska et al., 2013; Yaniv et al., 2006), complementing mammalian and cell culture models. In this review, we will summarize the use of zebrafish as a model system for lymphatic biology, compare the commonality and unique features of zebrafish lymphatic vessels, and provide perspectives on zebrafish as a unique model for lymphatic biology.

\section{LYMPHATIC SYSTEM: A BRIEF OVERVIEW}

Anatomical structures associated with the lymphatic system, particularly the lymph nodes, were first described as early as the era of Hippocrates (Chikly, 1997). However, it was not until $18^{\text {th }}$ century that the 'lymphatic system' was discovered (Chikly, 1997; Kerjaschki, 2014). Like blood vessels, lymphatic vessels display a highly stereotypic hierarchy. Lymphatic capillaries absorb excessive interstitial fluid within tissue and transport it to the larger caliber lymphatic vessels such as lymphatic collecting vessels and lymphatic trunks. While lymphatic capillaries usually lack muscular layers, therefore relying on peristaltic movement to transport lymph, larger lymphatic vessels do possess contractile muscular layers to facilitate forward movement of lymph (Dejana et al., 2009; Pfeiffer et al., 2008). However, compared to blood vessels, the muscular layers encompassing lymphatic vessels are generally thinner, which generate weaker contractile force. Therefore, lymph fluid within the large caliber lymphatic vessels can move in a retrograde manner. To prevent backflow of lymph, large caliber lymphatic vessels have lymphatic valves alongside the lymphatic wall, similar to the valves found in the large veins (Bazigou and Makinen, 2013). Excessive interstitial fluid is returned to blood circulation via subclavian veins and thoracic ducts which complete the lymphatic circulation. In addition to modulating the level of interstitial fluid, the lymphatic system plays an essential role in immune responses: Lymph nodes, where immature immune cells reside, function as a filter for foreign immunogens (Alitalo, 2011). 


\section{A. Mouse Lymphatic Vessel Development}

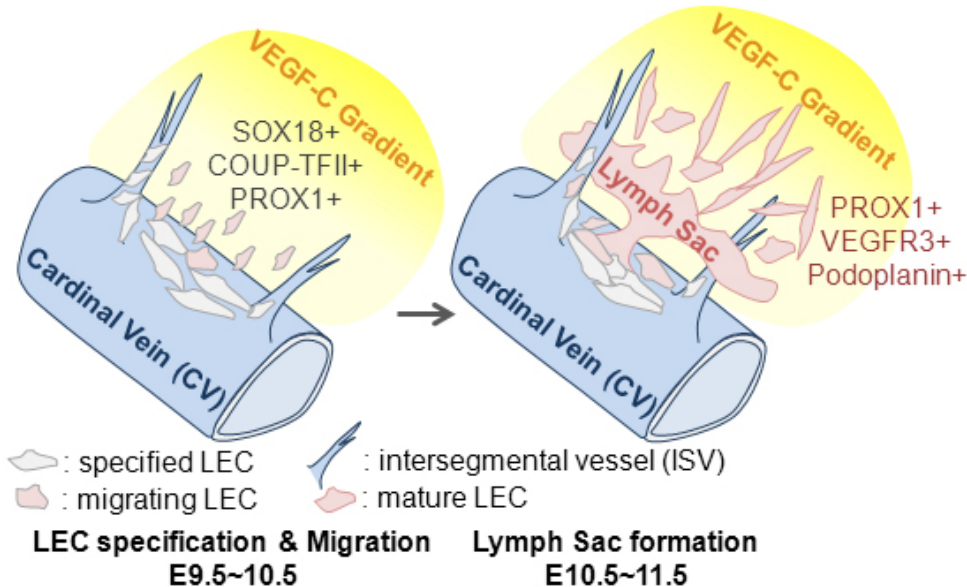

B. Zebrafish Lymphatic Vessel Development

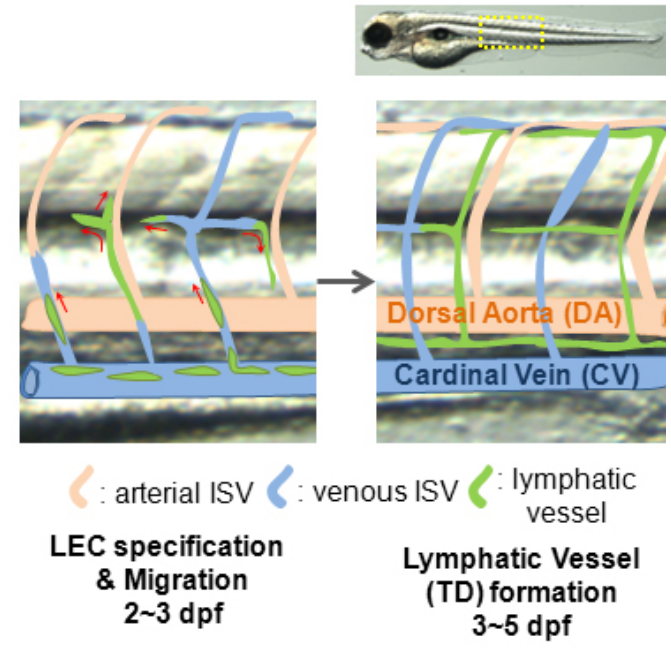

Fig. 1. Development of lymphatic vessels in mouse and zebrafish. (A) In mouse, individual LECs delaminate from the cardinal vein once the expression of lymphatic specific genes, including PROX1 and SOX18, initiates. Subsequently, LECs migrate toward the VEGF-C gradient and aggregate to form lymph sacs. (B) In zebrafish, LECs are thought to originate from the cardinal vein and a subset of venous intersegmental vessels during development. Although it is not clear how these LECs form lymphatic vessels, separate lymphatic vessels become visible at 3 dpf.

\section{EMERGENCE OF LYMPHATIC VESSELS DURING DEVELOPMENT IN MOUSE AND ZEBRAFISH}

The basic building blocks of lymphatic vessels, lymphatic endothelial cells, are known to emerge from blood endothelial cells (BECs) during development (Hägerling et al., 2013; Yang et al., 2012). While lymphatic endothelial cells (LEC) share certain molecular characteristics with BECs, cellular morphology of LECs appears to be distinct from BECs: For instance, both LECs and BECs strongly express PECAM and VE-Cadherin (Oliver and Srinivasan, 2010). However, LECs form button-like discontinuous junctions, which make lymphatic vessels highly permeable to excessive interstitial fluid, macromolecules, as well as migrating leukocytes, while BECs tend to form more organized and compact cell junctions (Neufeld et al., 2014).

During development, LECs, similar to other cell types found in cardiovascular systems of vertebrates, are thought to arise within the mesoderm (Marcelo et al., 2013). In particular, lateral plate mesoderm (LPM), a subset of the mesodermal layer in embryos, has been implicated as the developmental source of LECs. LECs first emerge around embryonic week 6 to 7 in humans, E10 in mice, and about 2 days post-fertilization (dpf) in zebrafish, only after the formation of blood vessels (Bruyère and Noël, 2010; Küchler et al., 2006; Neufeld et al., 2014; van der Putte, 1975). The majority of LECs appear to originate from veins during development (Fig. 1). At the turn of the $20^{\text {th }}$ century, Florence Sabin elegantly demonstrated that LECs originate from veins, which was validated by recent lineage tracing and in vivo time lapse imaging analyses in mouse, Xenopus, and zebrafish (Francois et al., 2011; Ny et al., 2005; Sabin, 1902; Srinivasan et al., 2007; Yaniv et al., 2006).

The earliest event during lymphatic development in mouse is the polarized expression of Lymphatic Vessel Endothelial Hyaluronan Receptor 1 (Lyve1) around E9 within a subset of ECs within the cardinal vein, which is the first sign of lymphatic competency of ECs (Banerji et al., 1999; Prevo et al., 2001). While the cardinal vein appears to be the major source of LECs, recent reports suggest that a significant number of LECs may be derived from ECs within the intersegmental vessels (ISVs) at approximately E10.5 and E11 (Yang et al., 2012). At E11.5, mature jugular lymph sacs are formed. During the specification and differentiation of LECs, the expression of SOX18, a member of SRY-related HMG-box (SOX) family of transcription factors, and venous enriched transcriptional factor Coup-TFII appear to be instrumental (François et al., 2008; Srinivasan et al., 2010): These two transcription factors cooperate to induce the expression of a lymphatic specific transcription factor, PROX1 (Wigle and Oliver, 1999; Wigle et al., 2002), which persists in LECs throughout their lifetime.

Although it is apparent that the PROX1 positive LECs within veins are the source of lymphatic vessels during development (Wigle et al., 2002), it is not clear how the separation between blood and lymphatic vessels occurs. Platelets have been implicated as the major driving force for initiating this process (Abtahian et al., 2003; Kim and Koh, 2010). By interacting with Podoplanin on the surface of LECs using CLEC-2 on their surface, platelets promote Syk/Spl76-mediated activation of Integrin signaling, which appears to be an essential step for the segregation of lymphatic vessels from the adjacent blood vessels. However, more recently, an alternative model has been proposed: Using high resolution in vivo imaging, Hägerling and colleagues suggest that individual PROX1 positive cells may delaminate from the cardinal vein, and subsequently aggregate to form the thoracic duct (Hägerling et al., 2013). Further analyses are warranted to resolve the potential discrepancy between these two models and to formulate the unified model. However, considering the high level of developmental, functional, and molecular heterogeneity of LECs (Oliver, 2004; Tammela and Alitalo, 2010), it is conceivable that these two mechanisms are not mutually exclusive, and collectively explain the complex nature of lymphatic development in vertebrates.

Similar to mouse, the progenitors for LECs appear to originate 


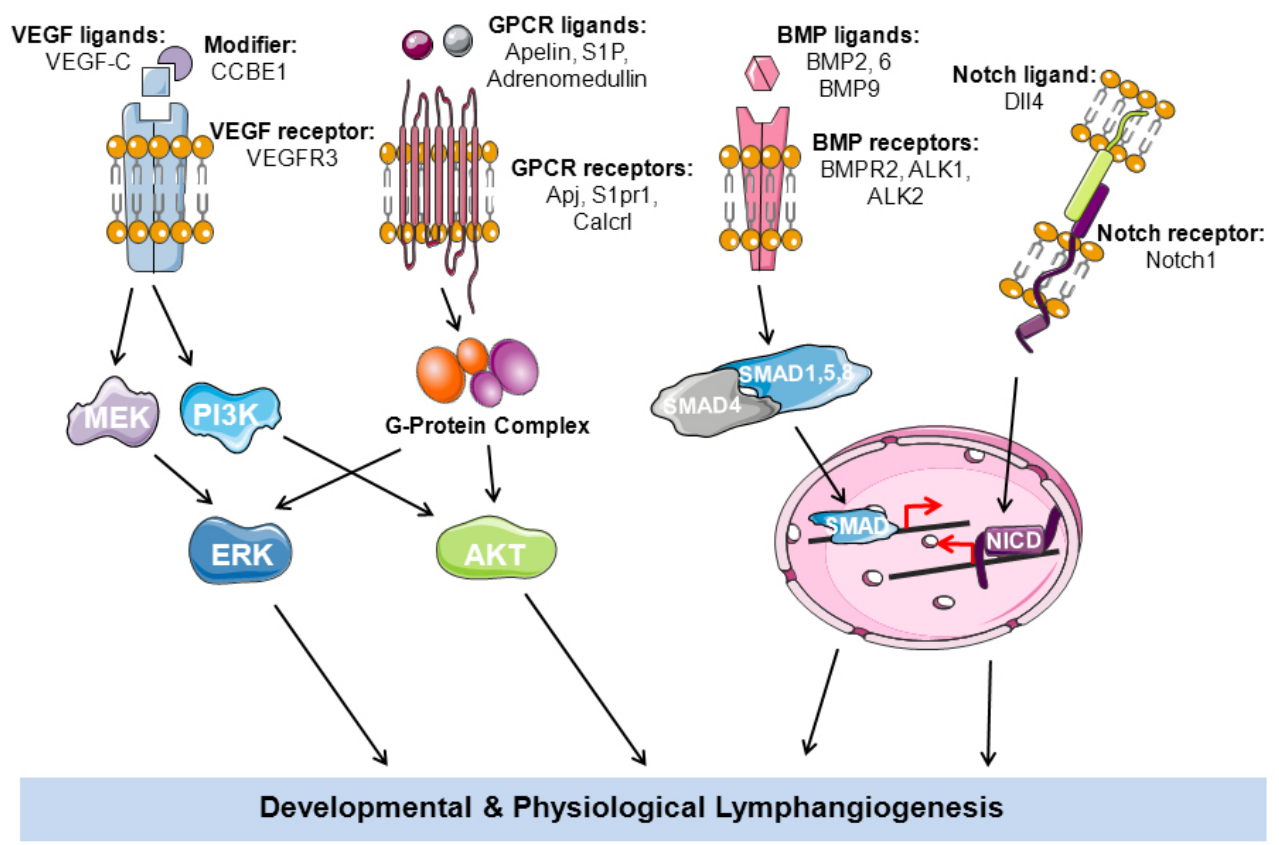

Fig. 2. Diverse signaling pathways modulate lymphatic development. To date, a number of signaling pathways have been implicated in lymphatic development. VEGF-C/VEGFR3 appears to be the most critical regulator of lymphatic development. In addition, the roles of other signaling pathways such as G Protein Coupled Receptor and BMP/TGF $\beta$ signaling, as well as Notch signaling pathways are known to modulate distinct aspects of lymphatic development.

from the cardinal vein around $2 \mathrm{dpf}$ in zebrafish (Küchler et al., 2006; Yaniv et al., 2006). Between 32 to $36 \mathrm{hpf}$, a group of angiogenic sprouts emerges from the cardinal vein, and eventually contribute to LECs. However, the exact anatomical locations of the progenitors for LECs remain largely unknown. The initial observation by Yaniv and colleagues indicate that LECs first migrate out of the parachodal vessel (Yaniv et al., 2006). However, this finding has been recently challenged by the fact that venous sprouts, which do not contribute to ISVs, may serve as a source for first group of LECs (Koltowska et al., 2013). Once specified, LECs rapidly migrate away from their original position and navigate along with arterial ISVs to make rudimentary lymphatic vessels (Yaniv et al., 2006). Around 3.5$4 \mathrm{dpf}$, the thoracic duct is clearly visible between the dorsal aorta and the cardinal vein (Kim and Kim, 2014; Yaniv et al., 2006), which expands and form lymph sacs at later stages (Okuda et al., 2012).

\section{MOLECULAR MECHANISMS MODULATING DEVELOPMENT OF THE LYMPHATIC VESSELS}

So far, a number of signaling pathways has been implicated in modulating lymphatic development (Fig. 2). Arguably, the most important signaling pathway for lymphatic development is Vascula Endothelial Growth Factor-C (VEGF-C) signaling. VEGF-C, which serves as a ligand for Vascular Endothelial Growth Factor Receptor 2 (VEGFR2/FIk1) and Vascular Endothelial Growth Factor Receptor 3 (VEGFR3/FIt4), was first identified as a protein with significant homology to VEGF-A and VEGF-B (Eichmann et al., 1998; Enholm et al., 2001; Jeltsch et al., 1997; Kukk et al., 1996). VEGF-C appears to be the main chemoat- tractant cue for migrating LECs (Karkkainen et al., 2004). In development, VEGF-C is highly expressed in the mesenchymal cells adjacent to developing lymphatic vessels, consistent with its proposed role. Targeted deletion of Vegfc in mouse led to embryonic lethality around E15 due to defects in the cardiovascular system (Karkkainen et al., 2004). In Vegfc null mice, lymphatic vessels did not form, indicating the essential function of VEGF-C in lymphatic development. Similarly, attenuation of VEGFR3, the main receptor for VEGF-C, led to similar lymphatic defects (Irrthum et al., 2000; Karkkainen et al., 2000). Taken together, these observations indicate that the function of VEGFC/VEGFR3 signaling pathway appears to be critical for the early development of the lymphatic vessels. At later stages, the function of VEGF-C/NEGFR3 for LECs appears to be comparable to that of VEGF-ANEGFR2 for BECs (Lohela et al., 2003). VEGF-C/VEGFR3 signaling serves as the main pro-lymphangiogenic cue by promoting proliferation, migration, and survival of LECs (Enholm et al., 2001; Jeltsch et al., 1997). Similar to many secreted ligands, VEGF-C is also produced in a preproform, and secreted as a pro-VEGF-C, which needs to be processed to be fully active (Lee et al., 2005; McColl et al., 2003). A disintegrin and metalloprotease with thrombospondin motifs-3 (ADAMTS3) has been recently shown to mediate proteolytic cleavage of pro-VEGF-C (Jeltsch et al., 2014), and the process is facilitated by Collagen and calcium-binding EGF domain-containing protein 1 (CCBE1) (Bos et al., 2011; Jeltsch et al., 2014). In humans, mutations in VEGF-C, VEGFR3, and CCBE1 have been shown to cause hereditary lymphedemas (Brouillard et al., 2014): For instance, mutations in VEGFR3 cause Milroy's disease (Irrthum et al., 2000; Karkkainen et al., 2000), and mutations in CCBE1 lead to Hennekam lymphan- 

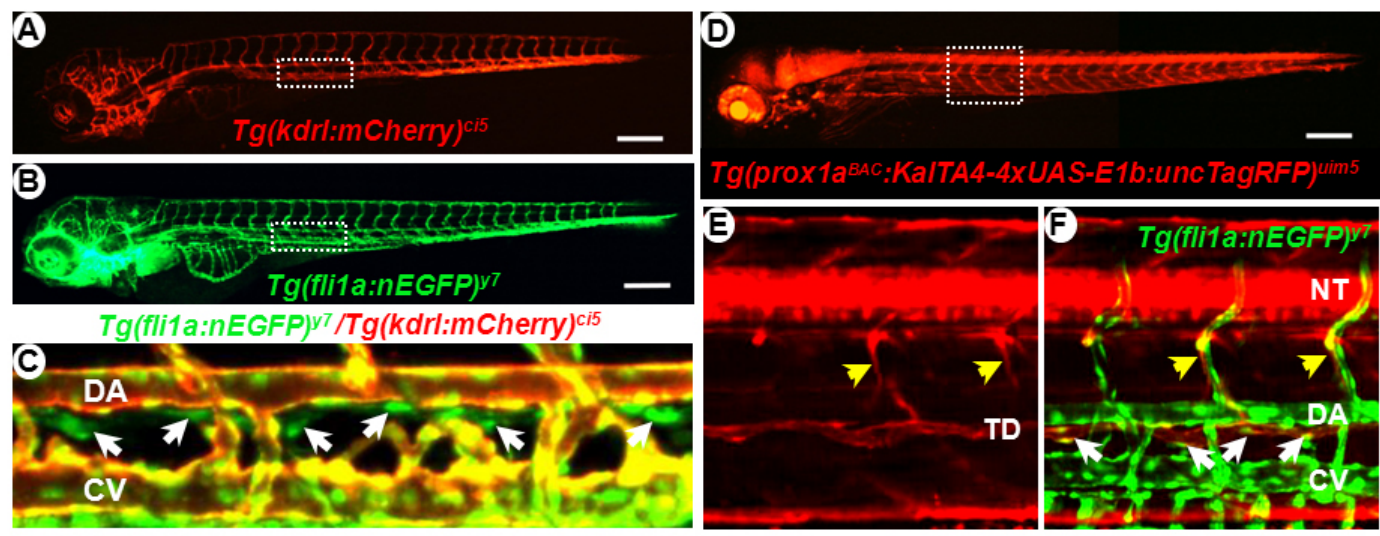

Fig. 3. Application of transgenic zebrafish for lymphatic research. Confocal images of 3dpf Tg(kdrl:mCherry) (A) and Tg(flia1:nEGFP) (B) Since $k d r l$ promoter is only active within BECs and fli1a promoter is active in both BECs and LECs, in the double transgenic line which carries both transgenes, BECs and LECs will be shown as yellow and green respectively. Area within the rectangle is shown in detail as (C). (D) Confocal image of $5 \mathrm{dpf} T g$ (prox1a:KalT;UAS: TagRFP). Area within the rectangle is shown in (E). (F) In combination with Tg(fli1a:nEGFP) transgenic line, the nucleus of individual LECs can be visualized and easily quantitated.

giectasia-lymphedema syndrome (Alders et al., 2009), further illustrating the importance of VEGF-C/VEGFR3 signaling in lymphatic development.

While VEGF-C/VEGFR3 signaling functions as an on/off switch for lymphatic development, additional signaling pathways modulate and fine-tune lymphatic development. For instance, it has been shown that a number of G-protein Coupled Receptors (GPCRs) are critical for lymphatic development (Karpinich and Caron, 2014). To date, at least four GPCR ligand/receptor pairs have been implicated; Adrenomedullin/RAMP/CALCRL (Fritz-Six et al., 2008; Karpinich et al., 2013), Sphingosine-1-phosphoate/EDG1 (Aoyagi et al., 2012; Yoon et al., 2008), CXCL12/CXCR4 (Cha et al., 2012), and Apelin/APJ (Kim et al., 2013). While diverse ligand and extracellular stimuli such as light and shear stress can activate GPCRs (Venkatakrishnan et al., 2013), virtually all GPCRs require trimeric Gprotein complexes, which consist of $\mathrm{G} \alpha, \mathrm{G} \beta$, and $\mathrm{G} \gamma$ subunits, to mediate their signaling activity and elicit cellular responses (Möhle and Drost, 2012; Smrcka, 2013). Within G-protein complexes, the $\mathrm{G} \alpha$ subunit generally determines the signaling outcomes of GPCRs (Gilman, 1987; Kimple et al., 2011). We have recently identified $\mathrm{G} \alpha 13$ as the main $\mathrm{G} \alpha$ subunit which functions as the essential downstream effector for diverse GPCRs to modulate lymphatic development (unpublished). GPCR signaling appears to be relayed by ERK1/2, AKT1/2 and ERK5 (Goldsmith and Dhanasekaran, 2007; Kidoya and Takakura, 2012; Nithianandarajah-Jones et al., 2012; Simon et al., 1991). Considering many targets of currently available pharmacological therapies are the components of GPCR signaling (DeWire and Violin, 2011; Dorsam and Gutkind, 2007; Smrcka, 2013), identification and understanding of the molecular and cellular basis of additional GPCRs and their contribution in lymphatic development and maintenance may help to develop effective translational applications for lymphatic disorders and associated diseases in the future.

While it is widely accepted that Notch signaling plays an instrumental role during angiogenesis, its function in lymphatic development remains unresolved because of conflicting evidence. For instance, lack of Notch signaling appears to be detrimental for lymphatic development; genetic deletion of
Notch1 or inhibition of Notch activity by DII4-Fc treatment led to lymphatic overgrowth and dilation of lymphatic vessels by promoting excessive sprouting of lymphatic vessels and increasing proliferation and survival of LECs (Murtomaki et al., 2013; Zheng et al., 2011), suggesting that Notch may negatively modulate lymphangiogenesis, similar to its role in angiogenesis. In contrast, Notch signaling appears to positively regulate lymphatic vessels in post-natal development (Niessen et al., 2011). Therefore, the function of Notch signaling during lymphatic development appears to be highly context-dependent. In addition to cell autonomous effects, Notch signaling is also critical for migration of lymphatic vessels. In zebrafish, attenuation of DII4, Notch1, or Notch6 compromise the extension of lymphatic vessels alongside the arterial ISVs, suggesting that Notch signaling may mediate the interaction between arterial and lymphatic vessels to guide migrating lymphatic vessels (Geudens et al., 2010).

Recently, bone morphogenetic protein (BMP) signaling has been implicated in lymphatic development. While antiangiogenic BMP9/Alk1 signaling promotes lymphatic development (Larrivée et al., 2012; Levet et al., 2013), pro-angiogenic BMP2/Alk2/3 signaling inhibits lymphatic development (Dunworth et al., 2013; Kim et al., 2012; Wiley et al., 2011), suggesting distinct cellular responses toward BMP signaling in BECs and LECs. In addition, transforming growth factor $\beta$ (TGF $\beta$ ), a signaling ligand related to BMPs, appears to provide contextdependent regulation of lymphatic development. While TGF $\beta$ signaling can substantially decrease proliferation and migration of human dermal lymphatic microvascular endothelial cells and negatively modulate lymphangiogenesis in vivo during regeneration (Oka et al., 2008), recent evidence suggest that TGF $\beta$ is indispensable for lymphangiogenesis in dermal lymphatic vessels (James et al., 2013).

In addition to the aforementioned signaling pathways, a number of structural proteins and signaling mediators appear to provide essential regulation during morphogenesis of the lymphatic vessels. In humans, mutations in gap junction proteins including Connexin 37 (CX37/GJA4), 43 (CX43/GJA1) and 47 (CX47/GJC2), Integrin A9 (ITGA9), and components of RAS signaling are found to predispose the propensity to develop 
lymphedema [reviewed in (Brouillard et al., 2014)]. Connexin 37 (CX37/GJA4), 43 (CX43/GJA1) and 47 (CX47/GJC2) are required for the formation of lymphatic valve leaflets in conjunction with Calcineurin signaling (Meens et al., 2014). Similarly, Integrin A9 (ITGA9) has been also implicated in the formation of lymphatic valve leaflets (Bazigou et al., 2009).

\section{AN OLD MODEL WITH A NEW TRICK?: ZEBRAFISH AS AN EMERGING MODEL FOR LYMPHATIC RESEARCH}

Since the first description of the lymphatic vessels in zebrafish less than a decade ago (Yaniv et al., 2006), zebrafish have become an emerging model system for lymphatic research. Many key attributes make zebrafish an attractive model system for studying the lymphatic system. First, zebrafish embryos are externally fertilized and transparent which allows for easy access and manipulations. One of the key challenges in investigating the early phases of lymphatic development in the traditional mouse model is in vivo real time imaging to track the movement of individual LECs over development since mouse embryos develop in utero. Although recent advances allow visualization of developing LECs in mouse embryos, this technique requires specific microscopes and complex preparations (Pollmann et al., 2014). Therefore, zebrafish can complement the mouse model and offers a unique advantage to unravel cellular behaviors during early lymphatic development. Second, like mice, zebrafish are a genetically tractable model with various transgenic lines which help to visualize and track individual cells during development (Ellertsdóttir et al., 2010; Kamei et al., 2010). For instance, there are a number of transgenic lines which selectively label developing LECs, each of which offers distinct advantages over others (Koltowska et al., 2013; Okuda et al., 2012). Third, traditional techniques for investigating lymphatic biology, such as lymphangiography, are applicable to zebrafish embryos (Yaniv et al., 2006)

\section{AVAILABLE TOOLS TO INVESTIGATE LYMPHATIC BIOLOGY IN ZEBRAFISH}

Diverse tools to manipulate and visualize the lymphatic vessels in zebrafish contribute to its value as the new model system in lymphatic research.

Transient and permanent genetic manipulation in zebrafish embryos

To delineate the function of a specific locus during development in zebrafish, antisense Morpholino oligonucleotides (MOs) are widely used (Ekker, 2000). MOs are 20 to 25 base pair long nucleotides which bind to the target sequence on pre-mature or mature mRNA to block translation or splicing (Bill et al., 2009). The translation blocking-MOs target the area encompassing the initiation codon of the mature mRNA and inhibit translation, while splice blocking-MOs bind to the splicing donor within the intron or splicing receptor within the exon in pre-mature mRNAs to block proper mRNA splicing, which in turn introduce a premature stop codon to generate non-functional proteins. By targeting existing mRNAs, translation blocking-MOs can interfere with both maternal and zygotic transcripts. In contrast, splice blocking-MOs can only inhibit zygotic transcripts since the maternal mRNA are fully processed transcripts, therefore lacking exon/intron boundaries. MOs can be directly injected into zebrafish embryos at 1-2 cell stages. Similar to an allelic series in classical mutagenesis, a range of phenotypes can be created by titrating the amount of injected $\mathrm{MO}$, which helps to define the function of a specific locus (Bill et al., 2009; Corey and Abrams, 2001; Heasman, 2002). Moreover, MOs can be combined with chemical antagonists/agonists, which allows for a small molecule screen in a sensitized background to identify modulators of lymphatic biology (Kim et al., 2013).

In addition, recent advances in targeted mutagenesis techniques allow fast and relatively easy generation of mutations affecting specific loci (Blackburn et al., 2013; Campbell et al., 2013). For instance, Zinc Finger Nucleases (ZFN) (Doyon et al. 2008; Meng et al., 2008), Transcription activator-like effector nucleases (TALEN) (Bedell et al., 2012), and more recently CRISPR/Cas9-based gene knockout (Hwang et al., 2013) strategies have been shown to be successful in generating mutations.

\section{Visualizing lymphatic vessels in zebrafish embryos} Lymphangiography (lymphography) is a traditional method to visualize the lymphatic vessels and diagnose abnormalities of the lymphatic system in the human body (Guermazi et al., 2003). In zebrafish, a modified version of lymphangiography can be performed to trace lymphatic structures. While injection of a radioactive contrasting agent followed by X-ray imaging is the standard procedure in humans (Guermazi et al., 2003), injection of fluorescently-labeled macromolecules such as Rhodamine dextran (e.g. $2 \times 10^{6} \mathrm{MW}$ ), is shown to be sufficient to visualize lymphatic vessels in zebrafish (Kim et al., 2013; Yaniv et al., 2006). In general, fluorescent spheres which are conjugated with high molecular weight particles, are injected subcutaneously into the tail region or directly into the thoracic duct (TD) in the trunk region of 3-5 dpf embryos or adults (Yaniv et al., 2006). In conjunction with chemical screening or MOmediated knock-down, lymphangiography allows rapid detection of structural deviations in lymphatic vessels.

In addition, transgenic lines can be used to visualize various vascular structures in zebrafish (Kamei et al., 2010; Wilkinson and van Eeden, 2014) (Fig. 3). Transgenic lines which express fluorescent reporters under vascular specific promoters such as vegfr2/kdrl (Jin et al., 2005), fli1a (Lawson and Weinstein, 2002) and etv2/etsrp (Kohli et al., 2013), have been generated. More recently, two lymphatic specific transgenic lines have been generated which express fluorescent reporters under prox1a or lyve1 promoters (Dunworth et al., 2013; Okuda et al., 2012).

\section{SIMILARITIES AND DIFFERENCES BETWEEN MAMMALIAN AND ZEBRAFISH MODELS}

While zebrafish is an attractive model to investigate lymphatic development, there are a number of pending issues to be addressed. For instance, it is possible that the molecular and cellular basis of lymphatic development and physiology in zebrafish may not be readily applicable to humans. Consistent with this idea, it has recently been suggested that zebrafish Prox1 is dispensable for lymphatic development (van Impel et al., 2014). By generating targeted mutagenesis, van Impel and colleague generated zebrafish prox 1 and prox $1 b$ double mutants, which have no discernible defects in lymphatic development. While intriguing, a number of issues need to be resolved to draw such a conclusion: First, both prox 1 and prox $1 b$ are maternally deposited transcripts. Even homozygous mutant embryos from the heterozygous mother will display an attenuated phenotype as shown in other mutations affecting loci whose transcripts are maternally provided (Abrams and Mullins, 2009; Lindeman and Pelegri, 2010; Wagner et al., 2004). 
Second, the zebrafish genome has yet another prox1-related gene, prox $11 /$ prox2, which is ubiquitously expressed. It is conceivable that Prox11/Prox2 may compensate for the lack of Prox1/Prox1b activity given their high sequence similarities (Pistocchi et al., 2008). Therefore, it is still premature to conclude that Prox 1 in zebrafish has a divergent role in lymphatic development compared to their homoeothermic relatives. However, considering that zebrafish is a poikilotherm, physiology of zebrafish may be distinct from that of homeotherms. Even with potential limitations, there is no doubt that zebrafish offer a unique opportunity which cannot be achieved by other models, and continues to thrive as a model to unlock secrets of lymphatic vessels.

\section{ACKNOWLEDGMENTS}

The authors thank the members of the Jin laboratory. This study was supported by grants from the NIH to S.-W. J. (HL090960 and HL119345). Figure 2 was generated in part using images from Servier Medical Art (http://www.servier.com/ Powerpoint-image-bank).

\section{REFERENCES}

Abrams, E.W., and Mullins, M.C. (2009). Early zebrafish development: it's in the maternal genes. Curr. Opin. Genet. Dev. 19, 396-403.

Abtahian, F., Guerriero, A., Sebzda, E., Lu, M.M., Zhou, R., Mocsai, A., Myers, E.E., Huang, B., Jackson, D.G., Ferrari, V.A., et al. (2003). Regulation of blood and lymphatic vascular separation by signaling proteins SLP-76 and Syk. Science 299, 247-251.

Achen, M.G., McColl, B.K., and Stacker, S.A. (2005). Focus on lymphangiogenesis in tumor metastasis. Cancer Cell 7, 121-127.

Alders, M., Hogan, B.M., Gjini, E., Salehi, F., Al-Gazali, L., Hennekam, E.A., Holmberg, E.E., Mannens, M.M., Mulder, M.F., Offerhaus, G.J., et al. (2009). Mutations in CCBE1 cause generalized lymph vessel dysplasia in humans. Nat. Genet. 41, 12721274.

Alitalo, K. (2011) The lymphatic vasculature in disease. Nat. Med. $17,1371-1380$.

Aoyagi, T., Nagahashi, M., Yamada, A., and Takabe, K. (2012). The role of sphingosine-1-phosphate in breast cancer tumorinduced lymphangiogenesis. Lymphat. Res. Biol. 10, 97-106.

Banerji, S., Ni, J., Wang, S.X., Clasper, S., Su, J., Tammi, R., Jones, M., and Jackson, D.G. (1999). LYVE-1, a new homologue of the CD44 glycoprotein, is a lymph-specific receptor for hyaluronan. J. Cell Biol. 144, 789-801.

Bazigou, E., and Makinen, T. (2013). Flow control in our vessels: vascular valves make sure there is no way back. Cell. Mol. Life Sci. 70, 1055-1066.

Bazigou, E., Xie, S., Chen, C., Weston, A., Miura, N., Sorokin, L., Adams, R., Muro, A.F., Sheppard, D., and Makinen, T. (2009). Integrin-alpha9 is required for fibronectin matrix assembly during lymphatic valve morphogenesis. Dev. Cell 17, 175-186.

Bedell, V.M., Wang, Y., Campbell, J.M., Poshusta, T.L., Starker, C.G., Krug, R.G., Tan, W., Penheiter, S.G., Ma, A.C., Leung, A.Y., et al. (2012). In vivo genome editing using a high-efficiency TALEN system. Nature 491, 114-118.

Bill, B.R., Petzold, A.M., Clark, K.J., Schimmenti, L.A., and Ekker, S.C. (2009). A primer for morpholino use in zebrafish. Zebrafish 6, 69-77.

Blackburn, P.R., Campbell, J.M., Clark, K.J., and Ekker, S.C. (2013). The CRISPR system--keeping zebrafish gene targeting fresh. Zebrafish 10, 116-118.

Bos, F.L., Caunt, M., Peterson-Maduro, J., Planas-Paz, L., Kowalski, J., Karpanen, T., van Impel, A., Tong, R., Ernst, J.A., Korving, J., et al. (2011). CCBE1 is essential for mammalian lymphatic vascular development and enhances the lymphangiogenic effect of vascular endothelial growth factor-C in vivo. Circ. Res. 109, 486491.

Brouillard, P., Boon, L., and Vikkula, M. (2014). Genetics of lymphatic anomalies. J. Clin. Invest. 124, 898-904.

Bruyère, F., and Noël, A. (2010). Lymphangiogenesis: in vitro and in vivo models. FASEB J. 24, 8-21.

Campbell, J.M., Hartjes, K.A., Nelson, T.J., Xu, X., and Ekker, S.C. (2013). New and TALENted genome engineering toolbox. Circ. Res. 113, 571-587.

Cha, Y.R., Fujita, M., Butler, M., Isogai, S., Kochhan, E., Siekmann, A.F., and Weinstein, B.M. (2012). Chemokine signaling directs trunk lymphatic network formation along the preexisting blood vasculature. Dev. Cell 22, 824-836.

Chikly, B. (1997). Who discovered the lymphatic system. Lymphology $30,186-193$

Connell, F.C., Gordon, K., Brice, G., Keeley, V., Jeffery, S., Mortimer, P.S., Mansour, S., and Ostergaard, P. (2013). The classification and diagnostic algorithm for primary lymphatic dysplasia an update from 2010 to include molecular findings. Clin. Genet. 84, 303-314.

Corey, D.R., and Abrams, J.M. (2001). Morpholino antisense oligonucleotides: tools for investigating vertebrate development. Genome Biol. 2, REVIEWS1015.

Dejana, E., Tournier-Lasserve, E., and Weinstein, B.M. (2009). The control of vascular integrity by endothelial cell junctions: molecular basis and pathological implications. Dev. Cell 16, 209-221.

DeWire, S.M., and Violin, J.D. (2011). Biased ligands for better cardiovascular drugs: dissecting G-protein-coupled receptor pharmacology. Circ. Res. 109, 205-216.

Dorsam, R.T., and Gutkind, J.S. (2007). G-protein-coupled receptors and cancer. Nat. Rev. Cancer 7, 79-94.

Doyon, Y., McCammon, J.M., Miller, J.C., Faraji, F., Ngo, C., Katibah, G.E., Amora, R., Hocking, T.D., Zhang, L., Rebar, E.J., et al. (2008). Heritable targeted gene disruption in zebrafish using designed zinc-finger nucleases. Nat. Biotechnol. 26, 702-708.

Dunworth, W.P., Cardona-Costa, J., Cagavi, E., Kim, J.D., Fischer, J.C., Meadows, S., Wang, Y., Cleaver, O., Qyang, Y., Ober, E.A., et al. (2013). Bone morphogenetic protein 2 signaling negatively modulates lymphatic development in vertebrate embryos. Circ. Res. 114, 56-66.

Eichmann, A., Corbel, C., Jaffredo, T., Bréant, C., Joukov, V., Kumar, V., Alitalo, K., and le Douarin, N.M. (1998). Avian VEGF-C cloning, embryonic expression pattern and stimulation of the differentiation of VEGFR2-expressing endothelial cell precursors. Development 125, 743-752.

Ekker, S.C. (2000). Morphants: a new systematic vertebrate functional genomics approach. Yeast 17, 302-306.

Ellertsdóttir, E., Lenard, A., Blum, Y., Krudewig, A., Herwig, L., Affolter, M., and Belting, H.G. (2010). Vascular morphogenesis in the zebrafish embryo. Dev. Biol. 341, 56-65.

Enholm, B., Karpanen, T., Jeltsch, M., Kubo, H., Stenback, F., Prevo, R., Jackson, D.G., Yla-Herttuala, S., and Alitalo, K. (2001) Adenoviral expression of vascular endothelial growth factor-C induces lymphangiogenesis in the skin. Circ. Res. 88, 623-629.

François, M., Caprini, A., Hosking, B., Orsenigo, F., Wilhelm, D., Browne, C., Paavonen, K., Karnezis, T., Shayan, R., Downes, M., et al. (2008). Sox18 induces development of the lymphatic vasculature in mice. Nature 456, 643-647.

Francois, M., Harvey, N.L., and Hogan, B.M. (2011). The transcriptional control of lymphatic vascular development. Physiology (Bethesda) 26, 146-155

Fritz-Six, K.L., Dunworth, W.P., Li, M., and Caron, K.M. (2008). Adrenomedullin signaling is necessary for murine lymphatic vascular development. J. Clin. Invest. 118, 40-50.

Geudens, I., Herpers, R., Hermans, K., Segura, I., Ruiz de Almodovar, C., Bussmann, J., De Smet, F., Vandevelde, W., Hogan, B.M., Siekmann, A., et al. (2010). Role of delta-like-4/Notch in the formation and wiring of the lymphatic network in zebrafish. Arterioscler. Thromb. Vasc. Biol. 30, 1695-1702.

Gilman, A.G. (1987). G proteins: transducers of receptor-generated signals. Annu. Rev. Biochem. 56, 615-649.

Goldsmith, Z.G., and Dhanasekaran, D.N. (2007). G protein regulation of MAPK networks. Oncogene 26, 3122-3142.

Gore, A.V., Monzo, K., Cha, Y.R., Pan, W., and Weinstein, B.M. (2012). Vascular development in the zebrafish. Cold Spring Harb. Perspect. Med. 2, a006684.

Guermazi, A., Brice, P., Hennequin, C., and Sarfati, E. (2003). Lymphography: an old technique retains its usefulness. Radiographics 23, 1541-1558; discussion 1559-1560.

Hägerling, R., Pollmann, C., Andreas, M., Schmidt, C., Nurmi, H. Adams, R.H., Alitalo, K., Andresen, V., Schulte-Merker, S., and 
Kiefer, F. (2013). A novel multistep mechanism for initial lymphangiogenesis in mouse embryos based on ultramicroscopy. EMBO J. 32, 629-644.

Heasman, J. (2002). Morpholino oligos: making sense of antisense? Dev. Biol. 243, 209-214.

Hwang, W.Y., Fu, Y., Reyon, D., Maeder, M.L., Tsai, S.Q., Sander, J.D., Peterson, R.T., Yeh, J.R., and Joung, J.K. (2013). Efficient genome editing in zebrafish using a CRISPR-Cas system. Nat. Biotechnol. 31, 227-229.

Irrthum, A., Karkkainen, M.J., Devriendt, K., Alitalo, K., and Vikkula, M. (2000). Congenital hereditary lymphedema caused by a mutation that inactivates VEGFR3 tyrosine kinase. Am. J. Hum. Genet. 67, 295-301.

James, J.M., Nalbandian, A., and Mukouyama, Y.S. (2013). TGF $\beta$ signaling is required for sprouting lymphangiogenesis during lymphatic network development in the skin. Development 140 3903-3914.

Jeltsch, M., Kaipainen, A., Joukov, V., Meng, X., Lakso, M., Rauvala, H., Swartz, M., Fukumura, D., Jain, R.K., and Alitalo, K. (1997). Hyperplasia of lymphatic vessels in VEGF-C transgenic mice. Science 276, 1423-1425.

Jeltsch, M., Jha, S.K., Tvorogov, D., Anisimov, A., Leppänen, V.M., Holopainen, T., Kivelä, R., Ortega, S., Kärpanen, T., and Alitalo, K. (2014). CCBE1 enhances lymphangiogenesis via ADAMTS3mediated VEGF-C activation. Circulation 129, 1962-1971.

Jin, S.W., Beis, D., Mitchell, T., Chen, J.N., and Stainier, D.Y. (2005). Cellular and molecular analyses of vascular tube and lumen formation in zebrafish. Development 132, 5199-5209.

Kamei, M., Isogai, S., Pan, W., and Weinstein, B.M. (2010). Imaging blood vessels in the zebrafish. Methods Cell Biol. 100, 27-54.

Karkkainen, M.J., Ferrell, R.E., Lawrence, E.C., Kimak, M.A., Levinson, K.L., McTigue, M.A., Alitalo, K., and Finegold, D.N. (2000). Missense mutations interfere with VEGFR-3 signalling in primary lymphoedema. Nat. Genet. 25, 153-159.

Karkkainen, M.J., Haiko, P., Sainio, K., Partanen, J., Taipale, J., Petrova, T.V., Jeltsch, M., Jackson, D.G., Talikka, M., Rauvala,

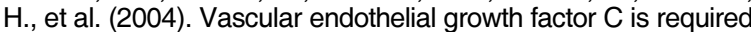
for sprouting of the first lymphatic vessels from embryonic veins. Nat. Immunol. 5, 74-80.

Karpinich, N.O., and Caron, K.M. (2014). Apelin signaling: new G protein-coupled receptor pathway in lymphatic vascular development. Arterioscler. Thromb. Vasc. Biol. 34, 239-241.

Karpinich, N.O., Kechele, D.O., Espenschied, S.T., Willcockson, H.H., Fedoriw, Y., and Caron, K.M. (2013). Adrenomedullin gene dosage correlates with tumor and lymph node lymphangiogenesis. FASEB J. 27, 590-600.

Kerjaschki, D. (2014). The lymphatic vasculature revisited. J. Clin. Invest. 124, 874-877.

Kidoya, H., and Takakura, N. (2012). Biology of the apelin-APJ axis in vascular formation. J. Biochem. 152, 125-131.

Kim, H., and Koh, G.Y. (2010). Platelets take the lead in lymphatic separation. Circ. Res. 106, 1184-1186.

Kim, J.D., and Kim, J. (2014). Alk3/Alk3b and Smad5 Mediate BMP signaling during lymphatic development in zebrafish. Mol. Cells $37,270-274$

Kim, J.D., Kang, H., Larrivée, B., Lee, M.Y., Mettlen, M., Schmid, S.L., Roman, B.L., Qyang, Y., Eichmann, A., and Jin, S.W. (2012). Context-dependent proangiogenic function of bone morphogenetic protein signaling is mediated by disabled homolog 2 . Dev. Cell 23, 441-448.

Kim, J.D., Kang, Y., Kim, J., Papangeli, I., Kang, H., Wu, J., Park, H., Nadelmann, E., Rockson, S.G., Chun, H.J., et al. (2013). Essential role of Apelin signaling during lymphatic development in zebrafish. Arterioscler. Thromb. Vasc. Biol. 34, 338-345.

Kimple, A.J., Bosch, D.E., Giguère, P.M., and Siderovski, D.P. (2011). Regulators of G-protein signaling and their $\mathrm{G} \alpha$ substrates: promises and challenges in their use as drug discovery targets. Pharmacol. Rev. 63, 728-749.

Kohli, V., Schumacher, J.A., Desai, S.P., Rehn, K., and Sumanas, S. (2013). Arterial and venous progenitors of the major axial vessels originate at distinct locations. Dev. Cell 25, 196-206.

Koltowska, K., Betterman, K.L., Harvey, N.L., and Hogan, B.M. (2013). Getting out and about: the emergence and morphogenesis of the vertebrate lymphatic vasculature. Development 140 , 1857-1870.

Küchler, A.M., Gjini, E., Peterson-Maduro, J., Cancilla, B., Wolburg,
H., and Schulte-Merker, S. (2006). Development of the zebrafish lymphatic system requires VEGFC signaling. Curr. Biol. 16, 1244-1248.

Kukk, E., Lymboussaki, A., Taira, S., Kaipainen, A., Jeltsch, M., Joukov, V., and Alitalo, K. (1996). VEGF-C receptor binding and pattern of expression with VEGFR-3 suggests a role in lymphatic vascular development. Development 122, 3829-3837.

Larrivée, B., Prahst, C., Gordon, E., del Toro, R., Mathivet, T. Duarte, A., Simons, M., and Eichmann, A. (2012). ALK1 signaling inhibits angiogenesis by cooperating with the Notch pathway. Dev. Cell 22, 489-500.

Lawson, N.D., and Weinstein, B.M. (2002). In vivo imaging of embryonic vascular development using transgenic zebrafish. Dev. Biol. 248, 307-318.

Lee, S., Jilani, S.M., Nikolova, G.V., Carpizo, D., and Iruela-Arispe, M.L. (2005). Processing of VEGF-A by matrix metalloproteinases regulates bioavailability and vascular patterning in tumors. $J$ Cell Biol. 169, 681-691.

Levet, S., Ciais, D., Merdzhanova, G., Mallet, C., Zimmers, T.A., Lee, S.J., Navarro, F.P., Texier, I., Feige, J.J., Bailly, S., et al. (2013). Bone morphogenetic protein 9 (BMP9) controls lymphatic vessel maturation and valve formation. Blood 122, 598-607.

Lindeman, R.E., and Pelegri, F. (2010). Vertebrate maternal-effect genes: Insights into fertilization, early cleavage divisions, and germ cell determinant localization from studies in the zebrafish. Mol. Reprod. Dev. 77, 299-313.

Lohela, M., Saaristo, A., Veikkola, T., and Alitalo, K. (2003). Lymphangiogenic growth factors, receptors and therapies. Thromb. Haemost. 90, 167-184.

Marcelo, K.L., Goldie, L.C., and Hirschi, K.K. (2013). Regulation of endothelial cell differentiation and specification. Circ. Res. 112, 1272-1287.

McColl, B.K., Baldwin, M.E., Roufail, S., Freeman, C., Moritz, R.L., Simpson, R.J., Alitalo, K., Stacker, S.A., and Achen, M.G. (2003). Plasmin activates the lymphangiogenic growth factors VEGF-C and VEGF-D. J. Exp. Med. 198, 863-868.

Meens, M.J., Sabine, A., Petrova, T.V., and Kwak, B.R. (2014) Connexins in lymphatic vessel physiology and disease. FEBS Lett. 588, 1271-1277.

Meng, X., Noyes, M.B., Zhu, L.J., Lawson, N.D., and Wolfe, S.A. (2008). Targeted gene inactivation in zebrafish using engineered zinc-finger nucleases. Nat. Biotechnol. 26, 695-701.

Möhle, R., and Drost, A.C. (2012). G protein-coupled receptor crosstalk and signaling in hematopoietic stem and progenitor cells. Ann. N Y Acad. Sci. 1266, 63-67.

Murdaca, G., Cagnati, P., Gulli, R., Spanò, F., Puppo, F., Campisi, C., and Boccardo, F. (2012). Current views on diagnostic approach and treatment of lymphedema. Am. J. Med. 125, 134140.

Murtomaki, A., Uh, M.K., Choi, Y.K., Kitajewski, C., Borisenko, V., Kitajewski, J., and Shawber, C.J. (2013). Notch1 functions as a negative regulator of lymphatic endothelial cell differentiation in the venous endothelium. Development 140, 2365-2376.

Neufeld, S., Planas-Paz, L., and Lammert, E. (2014). Blood and lymphatic vascular tube formation in mouse. Semin. Cell Dev. Biol. pii: S1084-9521(14)00025-1.

Niessen, K., Zhang, G., Ridgway, J.B., Chen, H., Kolumam, G., Siebel, C.W., and Yan, M. (2011). The Notch1-Dll4 signaling pathway regulates mouse postnatal lymphatic development Blood 118, 1989-1997.

Nithianandarajah-Jones, G.N., Wilm, B., Goldring, C.E., Müller, J., and Cross, M.J. (2012). ERK5: structure, regulation and function. Cell. Signal. 24, 2187-2196.

Ny, A., Koch, M., Schneider, M., Neven, E., Tong, R.T., Maity, S., Fischer, C., Plaisance, S., Lambrechts, D., Héligon, C., et al. (2005). A genetic Xenopus laevis tadpole model to study lymphangiogenesis. Nat. Med. 11, 998-1004.

Oka, M., Iwata, C., Suzuki, H.I., Kiyono, K., Morishita, Y., Watabe, T., Komuro, A., Kano, M.R., and Miyazono, K. (2008). Inhibition of endogenous TGF-beta signaling enhances lymphangiogenesis. Blood 111, 4571-4579.

Okuda, K.S., Astin, J.W., Misa, J.P., Flores, M.V., Crosier, K.E., and Crosier, P.S. (2012). Iyve1 expression reveals novel lymphatic vessels and new mechanisms for lymphatic vessel development in zebrafish. Development 139, 2381-2391.

Oliver, G. (2004). Lymphatic vasculature development. Nat. Rev. 
Immunol. 4, 35-45.

Oliver, G., and Srinivasan, R.S. (2010). Endothelial cell plasticity: how to become and remain a lymphatic endothelial cell. Development 137, 363-372.

Pfeiffer, F., Kumar, V., Butz, S., Vestweber, D., Imhof, B.A., Stein, J.V., and Engelhardt, B. (2008). Distinct molecular composition of blood and lymphatic vascular endothelial cell junctions establishes specific functional barriers within the peripheral lymph node. Eur. J. Immunol. 38, 2142-2155.

Pistocchi, A., Bartesaghi, S., Cotelli, F., and Del Giacco, L. (2008). Identification and expression pattern of zebrafish prox2 during embryonic development. Dev. Dyn. 237, 3916-3920.

Pollmann, C., Hägerling, R., and Kiefer, F. (2014). Visualization of lymphatic vessel development, growth, and function. Adv. Anat. Embryol. Cell Biol. 214, 167-186.

Prevo, R., Banerii, S., Ferguson, D.J., Clasper, S., and Jackson, D.G. (2001). Mouse LYVE-1 is an endocytic receptor for hyaluronan in lymphatic endothelium. J. Biol. Chem. 276, 1942019430.

Sabin, F.R. (1902). On the origin of the lymphatic system from the veins and the development of the lymph hearts and thoracic duct in the pig. Am. J. Anat. 1, 367-389.

Simon, M.I., Strathmann, M.P., and Gautam, N. (1991). Diversity of G proteins in signal transduction. Science 252, 802-808.

Smrcka, A.V. (2013). Molecular targeting of $\mathrm{G} \alpha$ and $\mathrm{G} \beta \gamma$ subunits: a potential approach for cancer therapeutics. Trends Pharmacol. Sci. 34, 290-298.

Srinivasan, R.S., Dillard, M.E., Lagutin, O.V., Lin, F.J., Tsai, S., Tsai, M.J., Samokhvalov, I.M., and Oliver, G. (2007). Lineage tracing demonstrates the venous origin of the mammalian lymphatic vasculature. Genes Dev. 21, 2422-2432.

Srinivasan, R.S., Geng, X., Yang, Y., Wang, Y., Mukatira, S., Studer, M., Porto, M.P., Lagutin, O., and Oliver, G. (2010). The nuclear hormone receptor Coup-TFIl is required for the initiation and early maintenance of Prox1 expression in lymphatic endothelial cells. Genes Dev. 24, 696-707.

Tammela, T., and Alitalo, K. (2010). Lymphangiogenesis: Molecular mechanisms and future promise. Cell 140, 460-476.

van der Putte, S.C. (1975). The development of the lymphatic system in man. Adv. Anat. Embryol. Cell Biol. 51, 3-60.

van Impel, A., Zhao, Z., Hermkens, D.M., Roukens, M.G., Fischer, J.C., Peterson-Maduro, J., Duckers, H., Ober, E.A., Ingham, P.W., and Schulte-Merker, S. (2014). Divergence of zebrafish and mouse lymphatic cell fate specification pathways. Development $141,1228-1238$
Venkatakrishnan, A.J., Deupi, X., Lebon, G., Tate, C.G., Schertler, G.F., and Babu, M.M. (2013). Molecular signatures of G-proteincoupled receptors. Nature 494, 185-194.

Wagner, D.S., Dosch, R., Mintzer, K.A., Wiemelt, A.P., and Mullins, M.C. (2004). Maternal control of development at the midblastula transition and beyond: mutants from the zebrafish II. Dev. Cell 6 , 781-790.

Wang, Y., and Oliver, G. (2010). Current views on the function of the lymphatic vasculature in health and disease. Genes Dev. 24, 2115-2126.

Wigle, J.T., and Oliver, G. (1999). Prox1 function is required for the development of the murine lymphatic system. Cell 98, 769-778.

Wigle, J.T., Harvey, N., Detmar, M., Lagutina, I., Grosveld, G., Gunn, M.D., Jackson, D.G., and Oliver, G. (2002). An essentia role for Prox1 in the induction of the lymphatic endothelial cell phenotype. EMBO J. 21, 1505-1513.

Wiley, D.M., Kim, J.D., Hao, J., Hong, C.C., Bautch, V.L., and Jin, S.W. (2011). Distinct signalling pathways regulate sprouting angiogenesis from the dorsal aorta and the axial vein. Nat. Cell Biol. 13, 686-692.

Wilkinson, R.N., and van Eeden, F.J. (2014). The zebrafish as a model of vascular development and disease. Prog. Mol. Biol. Transl. Sci. 124, 93-122.

Yang, Y., García-Verdugo, J.M., Soriano-Navarro, M., Srinivasan, R.S., Scallan, J.P., Singh, M.K., Epstein, J.A., and Oliver, G. (2012). Lymphatic endothelial progenitors bud from the cardinal vein and intersomitic vessels in mammalian embryos. Blood 120 2340-2348

Yaniv, K., Isogai, S., Castranova, D., Dye, L., Hitomi, J., and Weinstein, B.M. (2006). Live imaging of lymphatic development in the zebrafish. Nat. Med. 12, 711-716.

Yoon, C.M., Hong, B.S., Moon, H.G., Lim, S., Suh, P.G., Kim, Y.K. Chae, C.B., and Gho, Y.S. (2008). Sphingosine-1-phosphate promotes lymphangiogenesis by stimulating S1P1/Gi/PLC/Ca2+ signaling pathways. Blood 112, 1129-1138.

Zheng, W., Tammela, T., Yamamoto, M., Anisimov, A., Holopainen, T., Kaijalainen, S., Karpanen, T., Lehti, K., Ylä-Herttuala, S., and Alitalo, K. (2011). Notch restricts lymphatic vessel sprouting induced by vascular endothelial growth factor. Blood 118, 11541162.

Zuther, J.E., and Norton, S. (2012). Lymphedema management: the comprehensive guide for practitioners (Thieme, New York). 